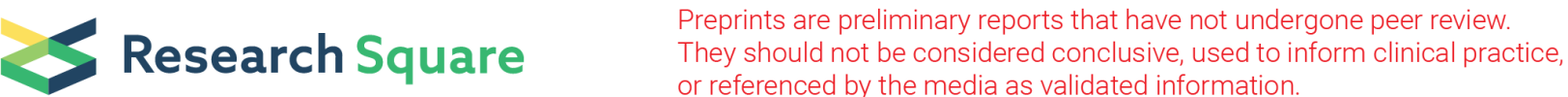

\section{Assessment of gingival inflammation indexes and matrix metalloproteinase (MMP-9) in patients with polycystic ovarian syndrome compared to control group}

\section{Avideh Maboudi}

Mazandaran University of Medical Sciences

Sara Shafizade ( $\sim$ sargol_shafizade@yahoo.com )

Mazandaran University of Medical Sciences

Fatemeh Nasiriamiri

Babol University of Medical Science https://orcid.org/0000-0001-6794-7627

Dourdi Qujeq

Babol University of Medical Science

Reza Ali Mohammadpour

Mazandaran University of Medical Sciences

Amirhosain Moaddabi

Mazandaran University of Medical Sciences

Mania Amiri

Babol University of Medical Science

Mehdi Gholami Bahnemiri

Babol University of Medical Science

\section{Sadra Yosefnia Pasha}

Babol University of Medical Science

Research article

Keywords: Gingivilis, Periodontal disease, Polycystic ovarian syndrome, Matrix metalloproteinase

Posted Date: August 28th, 2020

DOl: https://doi.org/10.21203/rs.3.rs-56300/v1

License: (1) This work is licensed under a Creative Commons Attribution 4.0 International License.

Read Full License 


\section{Abstract}

Background: There is emerging evidence to support the link between polycystic ovarian syndrome (PCOS) and periodontal disease, but the mechanisms associated with these two diseases have not been clearly elucidated but relate to different aspects of inflammation. This study aimed to determine the gingival inflammation indexes and matrix metalloproteinase-9 (MMP-9) in patients with polycystic ovarian syndrome compared to control group.

Methods: This is a case-control study in which 87 women referred to the Babol Clinic Private Hospital were divided into three groups: 26 women with PCOS diagnosed on the basis of Rotterdam criteria with gingivitis, 26 women with PCOS without gingivitis and 26 healthy women who were matched for age and body mass index. After recording the underlying variables, fasting saliva samples were taken from all participants in the morning before any periodontal intervention. The samples, which were transferred to Babol Molecular Cell Research Center under cold-chain conditions to measure the serum levels of MMP-9 using ELISA kits. Periodontal status was evaluated for Gingival index (GI), bleeding on probing (BOP), plaque index $(\mathrm{Pl})$. Analysis of variance was used to compare the results for the normal quantitative variables and Chi-square test was used to compare the qualitative variables. Significance level was considered less than 0.05 .

Results: The highest mean periodontal disease indexes (GI, BOP, PI) were observed in women with PCOS with gingivitis and then in women with PCOS without gingivitis and then in healthy women in control group $(p<0.05)$. The mean salivary MMP-9 levels were significantly higher in PCOS women $(388 / 37 \pm$ $75.05)$ compared to the control group (166/25 $\pm 35 / 43)$. Even in PCOS patients with healthy gingiva $(233.00 \pm 47.76)$ had higher levels of salivary MMP-9 than the control group and this difference was significant $(p<0.05)$

Conclusions: There is a positive association between periodontal disease and PCOS and that salivary MMP-9 levels are higher in PCOS women with and without gingivitis than healthy women. However, it is recommended that multicenter study with larger sample sizes are to be conducted to establish a clearer association.

\section{Introduction}

Polycystic ovary syndrome (PCOS) is one of the most common endocrinopathy, which affects $18 \%$ of pregnant women in their reproductive years $(1,2)$. The prevalence of this disorder in our country is 14.6 according to the Rotterdam criteria and $5.8 \%$ based on the National Institute of Health $(\mathrm{NIH})$ criteria (3). Due to its high prevalence and its complications, this syndrome places a financial burden on our healthcare system. It is estimated that annually PCOS in women aged 14 to 44 years costs $\$ 4.37$ billion to the U.S. economy (4). This disease is a multifactorial disorder, which is caused by the interaction of genetic and environmental factors (5). There are many evidences that suggest hypersensitivity and increased insulin resistance are the main pathophysiology of PCOS, which are followed by long-term 
health problems including diabetes, high blood pressure and cardiovascular disease (6). The exact etiology of PCOS is not quite clear, but it is a kind of hormonal imbalance accompanied by an increase in androgen and a decrease in reproductive function (7). On the other hand, because of chronic anovulation and exposure to estrogen without concomitant progesterone, there is an increased risk of uterine and cervical cancer (8). In addition to irregular menstruation, women with PCOS suffer from hirsutism (excessive growth of coarse hair in androgen-dependent areas) and infertility. Moreover, these women are at risk for some cardiovascular and metabolic diseases (9-11).

Because of the diversity of its signs and symptoms, PCOS is very difficult to diagnose (12). However, the Rotterdam criteria help identify and correctly diagnose it. According to these criteria, having at least two of the following three criteria is sufficient for diagnosis: anovulation (absence of ovulation) or oligoovulation (irregular ovulation), observation of a large number of small cysts in the ovaries (i.e., at least 12 or more small antral follicles on ultrasound scan of the ovaries), and presence of clinical or biochemical (or both) symptoms of increased androgen levels (13). The polycystic ovary syndrome is a common multifactor disorder in childbearing ages, which is identified by hyperandrogenism, primary ovarian insufficiency and polycystic ovarian morphology (14).

Periodontitis is a prevalent chronic infection caused by the inflammatory response initiated by the gum against the pathogenic microflora, which leads to the loss of alveolar bone and eventually the tooth (15). This process is resulted from the imbalance between the periodontal pathogens and the host response (16).

Gingivitis is a nondestructive inflammatory condition that occurs around the teeth (17). There are several evidences on the relationship between gingivitis and the systemic disorders including polycystic ovary syndrome, diabetes and cardiovascular disease (18). Since both gingivitis and PCOS are associated with systemic inflammation and insulin resistance, these two disorders might share a common pathophysiological pathway (19). Many studies suggest that chronic infections are followed by increased levels of reactive oxygen species, myeloperoxidase, oxidative stress, inflammatory cytokines (such as IL-6 and TNF-a), high-sensitivity C-reactive protein (hs-CRP), adhesion molecules, and blood lymphocytes and monocytes, a process which plays an important role in the etiology and pathogenesis of PCOS. This cascade of pro-inflammatory incidents is proposed as a potential link between periodontal disease and PCOS (20-22). In a study, that examined the levels of anti-inflammatory cytokines in gingival crevicular fluid (GCF), saliva and serum of women with PCOS and healthy women, it was shown that PCOS and gingivitis are synergistic with increased levels of IL-6 and TNF- $a .16$ (22). In another study, the level of matrix metalloproteinase-8 in the saliva and serum of patients with periodontal disease and PCOS showed an increase compared to the healthy controls (17). In some studies, the prevalence of periodontal disease was higher in patients with PCOS, which might be related to the role of chronic systemic inflammation in the pathophysiology of both PCOS and periodontal disease (24). However, in some other studies, there was no relationship between PCOS and inflammation markers such as CRP and IL-6 (25). Considering the contradictory results of the previous studies, the present study aims at investigating the 
indicators of gingival inflammation and matrix metalloproteinase-9 (MMP-9) in the saliva of patients with polycystic ovary syndrome compared to the control group.

\section{Materials And Method}

The present study was a case-control study conducted on women who were referred to the polycystic ovary syndrome clinic in Babol Clinic Private Hospital in north of Iran between 2018 and 2019. The case group was consisted of two groups of women with diagnosis of PCOS based on the Rotterdam criteria. One group included women who had gingivitis according to their periodontal examination and the other group included women who were healthy in this regard. The control group was consisted of systemically healthy women who were referred to the same center for screening for gynecological conditions. These women had normal menstrual cycles, had no clinical or biochemical signs of hyperandrogenism and their sonogram indicated the absence of polycystic ovaries. In case they had the same age and BMI (body mass index) as the case group and were periodontally healthy, they were added to the control group.

Using the ratio of means method and in line with the study of Durson et al. (26), the number of samples in each group (PCOS with gingivitis, PCOS without gingivitis and the periodontally and systemically healthy control group) was calculated to be 26 persons (total 78 ) with $95 \%$ confidence level and $90 \%$ power. The inclusion criteria for the case group were women aged 15 to 49 years who were diagnosed with PCOS based on the Rotterdam criteria which require any two out of three following criteria: anovulation or oligo-ovulation, PCO (volume of $>10 \mathrm{~mm} 3$ in at least one ovary in the sonogram, and clinical or biochemical sings of hyperandrogenism in the body (13). The inclusion criteria for the control group were healthy women with normal menstrual cycle, without clinical or biochemical signs of hyperandrogenism and no sign of PCO in their sonogram. The general inclusion criteria were: non-obese women $(\mathrm{BMI} \leq 30)$, non-smoker, non-drinker, no history of systemic conditions, no consumption of oral contraceptive pills in the past three months, and having a normal glucose tolerance test. The intervening variables included some drugs used during the past three to six months such as OCPs (oral contraceptive pills), antihypertensive drugs, steroid hormones, anti-inflammatory drugs and antibiotics. The exclusion criteria were diabetes, advanced kidney, liver and heart disease, hemorrhagic diseases in patients who were receiving periodontal treatment, having less than 20 teeth, pregnancy and smoking.

Among the women who were referred to gynecologic clinic at the Babol Clinic Private Hospital, those who were diagnosed with PCOS by gynecologist and also met the inclusion criteria were chosen for the case group and those who were referred to this center for screening tests and breast examination and were systemically healthy were sent to the school of dentistry at Babol University of Medical Sciences (the first executor of the present research plan) for evaluation of the following conditions:

1- Bleeding on Probing (BOP). 2- Plaque Index (PI) (Leo Index). 3- Gingival Index (Leo \& Silness). 4- The number of existing teeth.

The periodontal state of the participants was recorded as either healthy or with gingivitis. The person who was in charge of measuring the parameters did not know anything about the women's state - whether 
they were from the case group or the control group. Before the beginning of the study, the reliability of the measurement performed by the person in charge (student Sara Shafizade) was determined by calculating the correlation coefficient for the measurement of GI, PI and BOP. For this purpose, the named indices were measured twice in 5 patients with gingivitis with an interval of one week and in the end a correlation coefficient of more than 0.80 was acquired which indicated that the measurement had a good reliability. After receiving a written consent from the participants, the periodontal examination was performed in the case and the control group using the mirror and the periodontal probe (Williams probe) made by Medisporex (made in Pakistan). For this purpose, the Ramjford teeth including maxillary right first molar (tooth 16), maxillary left central incisor (tooth 11), maxillary left first bicuspid (tooth 24), mandibular left first molar (tooth 36), mandibular right central incisor (tooth 41) and mandibular right first bicuspid (tooth 44) were evaluated for GI, PI and BOP (picture 2.2). In case any of the Ramjford teeth were pulled out in the past, the adjacent tooth was evaluated (27). The diagnosis of periodontal disease was based on the presence of inflammation in the gum tissue and the periodontium. While in patients with gingivitis the gums turned red, became edematous and bled during procedures such as probing with periodontal probe, a healthy gum did not have these symptoms, were firm in consistency, appeared coral pink and did not bleed by probing. The participants' periodontal state was recorded as either healthy or with mild, moderate or severe gingivitis (54). After the periodontal examination, fasting saliva samples were taken from all participants in the morning before any periodontal intervention using the method proposed by Navazesh M (28). The samples, which were transferred to micro tubes that had already been thoroughly sterilized, were then sent to the Cellular and Molecular Biology Research Center at Babol University of Medical Sciences under cold-chain conditions to measure the serum levels of MMP-9 using ELISA kits. The data were collected based on the variables of the study including age, level of education, $\mathrm{BMI}$, degree of hirsutism or excessive hairiness (based on Ferriman-Gallwey scoring), infertility, length of menstrual cycle (minimum and maximum) and duration of bleeding.

The acquired data was analyzed by the SPSS20 software. The results of the three groups were compared using the analysis of the variance for normal quantitative variables and chi-square test for qualitative variables. If the gingivitis indices did not follow a normal distribution, the alternative non parametric test was performed. A p-value of less than 0.05 was considered to be statistically significant. The present study was approved by the research ethics committee of Mazandaran University of Medical Sciences in October 1, 2018 under the ethical approval code: IR.MAZUMS.REC.1397.2934.

\section{Results}

A total of 78 participants entered this study, who were divided into three different groups (26 participants in each group). Table 1 shows the demographic characteristics of the PCOS with gingivitis and PCOS without gingivitis and Healthy women. There was no statistically significant difference among these groups in terms of age, BMI, level of education and occupation $(p>0.05)$ and none of the participants were smokers. Regarding the systemic diseases, there were statistically significant differences among the three groups. $(p=0.02)$ While in the control group the women did not have any systemic diseases, in the PCOS with gingivitis group four of the women had thyroid disease and two of the women had 
hypertension and in the other case group (PCOS without gingivitis), one woman had thyroid disease and five women had hypertension. The Tukey's test revealed that there was a statistically significant difference between the control group and the two case groups, where six women from each of the groups mentioned that they had another systemic disease.

Table 1

Demographic characteristics of PCOS with gingivitis and PCOS without gingivitis and Healthy women (N $=87$ )

\begin{tabular}{|c|c|c|c|c|c|}
\hline \multicolumn{2}{|c|}{$\begin{array}{l}\text { Demographic } \\
\text { characteristics } \\
\text { (Mean } \pm \text { SD) }\end{array}$} & $\begin{array}{l}\text { PCOS with } \\
\text { gingivitis } \\
\text { (Mean } \pm \text { SD) }\end{array}$ & $\begin{array}{l}\text { PCOS without } \\
\text { gingivitis } \\
\text { (Mean } \pm \text { SD) }\end{array}$ & $\begin{array}{l}\text { Healthy } \\
\text { women } \\
\text { (Mean } \pm \text { SD) }\end{array}$ & $\begin{array}{l}\mathrm{P} \\
\text { value }\end{array}$ \\
\hline \multicolumn{2}{|l|}{ Age( year) } & $27.19 \pm 5.07$ & $26.96 \pm 5.66$ & $27.35 \pm 4.69$ & 0.96 \\
\hline \multicolumn{2}{|l|}{$\mathrm{BMI}(\mathrm{kg} / \mathrm{m} 2)$} & $24.94 \pm 2.93$ & $25.25 \pm 3.35$ & $26.52 \pm 3.33$ & 0.18 \\
\hline \multicolumn{2}{|l|}{$\mathrm{N}(\%)$} & $\mathrm{N}(\%)$ & $\mathrm{N}(\%)$ & $\mathrm{N}(\%)$ & \multirow[t]{4}{*}{0.36} \\
\hline \multirow[t]{3}{*}{ Education } & <Diploma & $4(15.4)$ & $1(3.8)$ & $4(15.4)$ & \\
\hline & Diploma & $9(34.6)$ & $10(38.5)$ & $13(50)$ & \\
\hline & $<$ Diploma & $9(34.6)$ & 15(57.7) & $13(50)$ & \\
\hline \multirow[t]{2}{*}{ occupation } & housewife & 23(88.5) & $22(84.6)$ & $24(92.23)$ & \multirow[t]{2}{*}{0.68} \\
\hline & Employed & $3(11.5)$ & $4(15.4)$ & $2(7.7)$ & \\
\hline
\end{tabular}

In terms of the family history of diabetes, the women in the control group did not have any history, but in the PCOS with gingivitis group six women and in the PCOS without gingivitis group four women had a family history of diabetes. However, the ANOVA results showed that the differences were not statistically different.

Regarding the signs and symptoms of PCOS, there were several cases of infertility, PCO appearance on ultrasound, higher degrees of hirsutism (based on Ferriman-Gallwey scoring), irregular menstruation, long menstrual cycles and menstrual bleeding periods of less than three days in the two case groups. But none of these signs were observed in the control group. The ANOVA results indicated that the differences among the three groups were statistically significant $(p=0.001)$. Moreover, the results of the Tukey's test revealed that there was a statistically significant difference between the control group and the two case groups $(p=0.001)$, however, there was no significant difference between the two case groups in this regard (Table 2). 
Table 2

Comparison of polycystic ovary syndrome indices in PCOS with gingivitis and PCOS without gingivitis and Healthy women $(\mathrm{N}=87)$

\begin{tabular}{|c|c|c|c|c|c|}
\hline \multicolumn{2}{|c|}{ Indicators of polycystic ovary syndrome } & $\begin{array}{l}\text { PCOS with } \\
\text { gingivitis } \\
\text { (Mean } \pm \text { SD) }\end{array}$ & $\begin{array}{l}\text { PCOS without } \\
\text { gingivitis } \\
\text { (Mean } \pm \text { SD) }\end{array}$ & $\begin{array}{l}\text { Healthy } \\
\text { women } \\
\text { (Mean } \pm \\
\text { SD) }\end{array}$ & $\begin{array}{l}\mathrm{P} \\
\text { value }\end{array}$ \\
\hline \multirow[t]{2}{*}{ Infertility } & has it & $21(80.8)$ & $14(53.8)$ & 0 & \multirow[t]{2}{*}{0.001} \\
\hline & does not have & $5(19.2)$ & $12(46.2)$ & $26(100)$ & \\
\hline \multirow{2}{*}{$\begin{array}{l}\text { View of PCO on } \\
\text { ultrasound }\end{array}$} & has it & $22(40.6)$ & $20(76.9)$ & 0 & \multirow[t]{2}{*}{0.001} \\
\hline & does not have & $4(15.4)$ & $6(23.1)$ & $26(100)$ & \\
\hline \multirow[t]{2}{*}{ Irregular menstruation } & has it & $9(34.6)$ & $14(53.8)$ & 0 & \multirow[t]{2}{*}{0.001} \\
\hline & does not have & $7(65.4)$ & $12(46.2)$ & $26(100)$ & \\
\hline \multirow[t]{2}{*}{$\begin{array}{l}\text { Menstrual cycle } \\
\text { duration }\end{array}$} & $\begin{array}{l}21 \text { to } 42 \text { days } \\
\text { (normal) }\end{array}$ & $2(46.2)$ & $14(53.8)$ & $26(100)$ & \multirow[t]{2}{*}{0.001} \\
\hline & $\begin{array}{l}\text { More than } 42 \\
\text { days }\end{array}$ & 14(53.8) & 12(46.2) & 0 & \\
\hline \multirow{3}{*}{$\begin{array}{l}\text { The length of the } \\
\text { menstrual period }\end{array}$} & normal & 15(57.7) & 18(69.2) & $26(100)$ & \multirow[t]{2}{*}{0.001} \\
\hline & abnormal & $11(42.3)$ & $8(30.8)$ & 0 & \\
\hline & & $($ Mean $\pm S D)$ & $($ Mean $\pm S D)$ & $\begin{array}{l}\text { (Mean } \pm \\
\text { SD) }\end{array}$ & \\
\hline \multicolumn{2}{|l|}{ Hirsutism score } & $3.58 \pm 2.06$ & $3.28 \pm 1.68$ & $\begin{array}{l}1.27 \pm \\
1.07\end{array}$ & 0.001 \\
\hline
\end{tabular}

The periodontal indices in the three study groups were compared using the ANOVA and the results indicated that there were statistically significant differences among the three groups in all items $(p<$ 0.001) (Table 3). Moreover, the results of the Tukey's HSD test, which was used for pairwise comparison of the periodontal indices between each two groups, showed that in some items the difference between the means were statistically significant (Table 4). 
Table 3

Mean and standard deviation of periodontal indices in PCOS with gingivitis and PCOS without gingivitis and healthy women $(\mathrm{N}=87)$

\begin{tabular}{|lllll|}
\hline periodontal indices & $\begin{array}{l}\text { PCOS with gingivitis } \\
\text { (Mean } \pm \text { SD) }\end{array}$ & $\begin{array}{l}\text { PCOS without gingivitis } \\
\text { (Mean } \pm \text { SD) }\end{array}$ & $\begin{array}{l}\text { Healthy women } \\
\text { (Mean } \pm \text { SD) }\end{array}$ & P value \\
\hline GI & $1.69 \pm 0.30$ & $0.12 \pm 0.38$ & $0.37 \pm 0.07$ & 0.001 \\
\hline PI & $1.54 \pm 0.57$ & $0.71 \pm 0.31$ & $0.33 \pm 0.10$ & 0.001 \\
\hline BOP & $\% 51 \pm 0.17$ & $\% 12 \pm 0.05$ & $\% 9 \pm 0.04$ & 0.001 \\
\hline
\end{tabular}


Table 4

pairwise comparison of the periodontal indices between each two groups

\begin{tabular}{|c|c|c|c|c|c|}
\hline Dependent Variable & (I) group & (J) group & Mean Difference (I-J) & Std. Error & Sig. \\
\hline \multirow[t]{6}{*}{ GI } & \multirow[t]{2}{*}{1} & 2 & $5.27585^{\star}$ & .21879 & .000 \\
\hline & & 3 & $5.28581^{*}$ & .21879 & .000 \\
\hline & \multirow[t]{2}{*}{2} & 1 & $-5.27585^{*}$ & .21879 & .000 \\
\hline & & 3 & .00996 & .21879 & .999 \\
\hline & \multirow[t]{2}{*}{3} & 1 & $-5.28581^{\star}$ & .21879 & .000 \\
\hline & & 2 & -.00996 & .21879 & .999 \\
\hline \multirow[t]{6}{*}{$\mathrm{PI}$} & \multirow[t]{2}{*}{1} & 2 & $3.29422^{*}$ & .42441 & .000 \\
\hline & & 3 & $4.81318^{*}$ & .42441 & .000 \\
\hline & \multirow[t]{2}{*}{2} & 1 & $-3.29422^{*}$ & .42441 & .000 \\
\hline & & 3 & $1.51896^{*}$ & .42441 & .002 \\
\hline & \multirow[t]{2}{*}{3} & 1 & $-4.81318^{*}$ & .42441 & .000 \\
\hline & & 2 & $-1.51896^{*}$ & .42441 & .002 \\
\hline \multirow[t]{6}{*}{ BOP } & \multirow[t]{2}{*}{1} & 2 & $1.52690^{*}$ & .12089 & .000 \\
\hline & & 3 & $1.66810^{*}$ & .12089 & .000 \\
\hline & \multirow[t]{2}{*}{2} & 1 & $-1.52690^{*}$ & .12089 & .000 \\
\hline & & 3 & .14120 & .12089 & .476 \\
\hline & \multirow[t]{2}{*}{3} & 1 & $-1.66810^{*}$ & .12089 & .000 \\
\hline & & 2 & -.14120 & .12089 & .476 \\
\hline
\end{tabular}

1. PCOS with gingivitis 2. PCOS without gingivitis 3 . healthy women

Table 5 presents the mean and the standard deviation of matrix metalloproteinase-9 (MMP-9) in the saliva of the women in each of the three groups. The differences among these groups were statistically significant. Tukey's HSD test was also performed for pairwise comparison between each two groups, which showed that women with PCOS had a higher level of MMP-9 in their saliva compared to the healthy women in the control group. Even the women in the PCOS without gingivitis group, who had a healthy gum, had a higher level of MMP-9 in their saliva compared to the women in the control group and 
the difference was statistically significant $(p=0.001)$. Table 6 shows the Pairwise comparison of salivary matrix metalloproteinase 9 between each two groups.

\section{Table 5}

Mean and standard deviation of saliva metalloproteinase-9 matrix in PCOS with gingivitis and PCOS without gingivitis and Healthy women $(\mathrm{N}=87)$

\begin{tabular}{|lllll|}
\hline MMP-9 & Mean \pm SD & \multicolumn{2}{l}{ 95\% C I } & P value \\
& & Lower Upper & \\
\hline PCOS with gingivitis & $388.36 \pm 75.05$ & 419.00 & 358.37 & 0.001 \\
\hline PCOS without gingivitis & $239.00 \pm 47.76$ & 258.29 & 219.71 & 0.001 \\
\hline Healthy women & $166.25 \pm 35.43$ & 180.56 & 151.93 & 0.001 \\
\hline
\end{tabular}

Table 6

Pairwise comparison of salivary matrix metalloproteinase-9 between each two groups

\begin{tabular}{|llllll|}
\hline MMP-9 & $\begin{array}{l}\text { PCOS with } \\
\text { gingivitis } \\
\text { (Mean } \pm \text { SE) }\end{array}$ & $\begin{array}{l}\text { PCOS without } \\
\text { gingivitis } \\
\text { (Mean } \pm \text { SE) }\end{array}$ & $\begin{array}{l}\text { Healthy } \\
\text { women }\end{array}$ & $\begin{array}{l}\text { P } \\
\text { value }\end{array}$ \\
\hline 1 & PCOS with gingivitis & & $149.68 \pm 15.33$ & $222.43 \pm$ & 0.001 \\
\hline 2 & $\begin{array}{l}\text { PCOS without } \\
\text { gingivitis }\end{array}$ & $149.68 \pm 15.33$ & & $72.75 \pm 15.33$ & 0.001 \\
\hline 3 & Healthy women & $222.43 \pm 15.33$ & & & 0.001 \\
\hline
\end{tabular}

\section{Discussion}

The results of the present study showed that after adjusting the BMI and age, the parameters of periodontal inflammation ( $\mathrm{GI}, \mathrm{PI}$ and $\mathrm{BOP}$ ) were higher in women with PCOS compared to healthy women $(p=0.001)$. Moreover, the salivary levels of MMP-9 were much higher in women with PCOS than in the control group. Even in those patients with PCOS who had a healthy gum the level of MMP-9 in saliva was higher compared to the control group. In accordance with the present study, Kellesarians et al. (2017) concluded in their review study that patients with PCOS had a higher prevalence of periodontal diseases which might be related to the role of chronic systemic inflammation in the pathophysiology of the two diseases (24). In the study of Dursun et al. (2011), the clinical periodontal parameters including probing depth (PD), plaque index (PI), gingival index (GI), bleeding on probing (BOP) and clinical attachment loss (CAL) were compared in 25 women with PCOS and 27 healthy women who were matched in terms of age and BMI. They stated that all of the evaluated periodontal parameters except the CAL were higher in patients with PCOS than in healthy participants in the control group. Moreover, the volume of the gingival crevicular fluid as well as the amount of nitric oxide (NO) and myeloperoxidase (MPO) in the gingival sulcus was higher among the patients with PCOS. Their findings suggested that the local/periodontal 
oxidant status in women with PCOS was damaged and their susceptibility for periodontal diseases increased considerably (26). Periodontal diseases are one of the most prevalent chronic inflammatory diseases (29). The relationship between periodontal and systemic diseases such as cardiovascular diseases (30), diabetes (31), stroke (32) and respiratory diseases (33) is quite acknowledged. The first time, the study of Kelly et al. in 2001 revealed the relationship between PCOS and chronic inflammatory diseases. In their study, after adjusting the effect of BMI, women with PCOS had higher concentrations of CRP, which is a marker for inflammation (34). Also, the study of Ozkaca et al. (2012), which compared three groups of women, reported that concentration of markers of inflammation such as interleukin- 6 in saliva and serum of women with PCOS and gingivitis was higher than women with PCOS who had a healthy gum. Moreover, the concentration of TNF-a in serum was higher in the two groups of women with PCOS compared to the control group. It appears that PCOS and gingivitis have a synergistic effect on the increase in inflammatory cytokines such as interleukin- 6 and TNF-a. However, they emphasized that further studies were needed to clarify the possible link between PCOS and periodontal diseases (35). On the other hand, the study of Mohling et al. (2004) stated that PCOS is not related to the increase in markers of chronic inflammation. In this study, 57 women with PCOS and 20 healthy volunteers, who were the same age and had the same BMI as the case group, were compared in terms of markers of inflammation including IL- 6 and CRP and parameters such as obesity, insulin resistance and hyperandrogenism. The results showed that plasma CRP and IL- 6 were not higher in two groups of women with PCOS (fat and thin women) compared to the control group (36). However, the recent studies have reported that women with PCOS have had chronic mild inflammation and this factor has been considered to have a role in increasing the risk of cardiovascular disease and type 2 diabetes (37-39).

Many studies showed that chronic infectious diseases are associated with elevated levels of reactive oxygen species, myeloperoxidase, oxidative stress, inflammatory cytokines (such as IL-6 and TNF- $\alpha$ ), hypersensitive reactive proteins, adhesion molecules, and blood lymphocytes and monocytes (20-22). PCOS is also associated with low-grade systemic inflammation, which is followed by an increase in multiple inflammatory markers such as C-reactive protein, interleukin-18, monocyte chemo-attractant protein-1 (MCP-1/CCL2), and white blood cells, as well as endothelial dysfunction and increased oxidative stress (40). This cascade of pro-inflammatory events has been suggested as a possible link between periodontal disease and PCOS, a process that may play a role in the etiology and pathogenesis of PCOS (20-22). However, studies are not in consistency with each other in this regard. In the study of Ekali (2017), there was no relationship between PCOS and markers of inflammation like CRP and IL-6 (25). For this reason, in a review study the researchers concluded that the number of curent studies on low-grade inflammatory biomarkers in patients with PCOS are limited and existing studies are heterogeneous. Therefore, they recommended further studies on inflammatory biomarkers in women with PCOS (40).

In the present study, the level of salivary MMP-9 in patients with PCOS was significantly higher than in the control group. The other finding of this study was that the level of salivary MMP-9 in patients with PCOS who had a healthy gum was higher than in the control group. Some researchers believe that MMPs might have a role in the pathogenesis of PCOS and the possible mechanism proposed is that the rate of growth 
and regeneration of ovarian follicles is modified by the balance between MMPs in the extracellular environment (41). The study of Ranjbaran et al. in 2016 was the first one on the involvement of the activity of MMP-9 in pathogenesis of PCOS. They showed that the serum level of MMP-9 was significantly higher in women with PCOS compared to the control group (42). The study of Dambala et al. in 2017 also showed the same result (43). In the study of Javed et al. (2913), the level of MMP-8 was higher in the saliva and serum of patients with PCOS and periodontal disease compared to the control group (comprised of women who were systemically healthy and had healthy gums) (16). However, the results of studies are contradictory (44-46).

MMPs are a family of proteolytic enzymes, which destroy the extracellular matrix and the basement membrane. That is the reason why they are important in physiological and pathological processes. MMP9 is a 92-kilodalton protein with protease activity whose main substrate is extracellular matrix and basement membrane tissue connections. It is the only member of the family that is able to attach and digest collagen as the most important composition of the basement membrane due to its 3 -fibronectin structure (47). MMPs are found in a variety of environments. So far, more than 26 types of these enzymes have been identified in humans that are structurally very similar. They have a very important role in migration of lymphoid and myeloid cells, physiological rearrangement of tissues including organogenesis, normal growth, embryonic growth, angiogenesis and ovulation (48). MMPs inhibit cancer cell apoptosis by various mechanisms including inhibition of NK-cell function (49). That is why increased plasma levels of MMP-9 have been observed in a variety of cancers such as gastric, breast, colon, lung, head and neck cancers (50).

\section{Conclusion}

Various studies have been conducted on the relationship between periodontal disease and polycystic ovary syndrome, most of which emphasize on the importance of the effect of periodontal diseases on polycystic ovary syndrome. However, there are conflicting results in these studies. The present study showed that the parameters of gingival inflammation ( $\mathrm{Gl}, \mathrm{PI}$, and BOP) were higher in women with PCOS compared to healthy women. To the best of our knowledge, there has been no other studies which compared the salivary level of MMP-9 in healthy women and women with PCOS and periodontal disease.

As a result, for the first time the present study showed that the levels of salivary MMP-9 were much higher in patients with PCOS and periodontal disease than in healthy women in the control group. Another finding of this study was that in PCOS patients who did not have periodontal disease, levels of salivary MMP-9 were higher than the control group (healthy women). According to the results of this study, it is assumed that there might be a positive association between periodontal diseases and PCOS, and that salivary levels of MMP-9 are higher in women with PCOS, who have or do not have gingivitis, than in healthy women. However, it is recommended that a study be conducted with a larger number of samples and in a multi-center manner to demonstrate the existence of this relationship more clearly and strongly.

\section{Limitations}


it is a sure thing that all studies have limitations. One of the limitations of this study was the limited number of women with polycystic ovary syndrome and the lack of cooperation of a number of patients to participate in periodontal examination which led to their exclusion from the study. Moreover, transfer and storage of samples in appropriate conditions was one of the challenges of this study. However, due to the proximity of the sampling site to the sample storage center, these problems decreased.

\section{List Of Abbreviations}

PCOS: polycystic ovarian syndrome

MMPs: Matrix metallopeptidases

MMP-9: matrix metalloproteinase-9

BMI: body mass index

hs-CRP: high-sensitivity C-reactive protein

Gl: Gingival index

BOP: bleeding on probing

PI: plaque index

ELISA: enzyme-linked immunosorbent assay

GCF: gingival crevicular fluid

CRP: C-reactive protein,

IL: interleukin

MCP-1: monocyte chemo-attractant protein-1

TNF- $a$ : Tumor necrosis factor- $a$

NO: nitric oxide

MPO: myeloperoxidase

OCPs: Oral contraceptive pills

MUMS: Mazandaran University of Medical Sciences,

\section{Declarations}


All procedures were carried out on the basis of ethics standards of the responsible committee on human experimentation (institutional and national) and to the Declaration of Helsinki. In addition, the ethics Committee of MUMS approved the study (code: IR.MAZUMS.REC.1397.2934.). All participants provided an informed written and signed consent form. All patients provided written informed consent.

\section{Consent for publication:}

We confirm that the manuscript entitled "Assessment of gingival inflammation indexes and matrix metalloproteinase (MMP-9) in patients with polycystic ovarian syndrome compared to control group" has been read and approved by all named authors and that there are no other persons who satisfied the criteria for authorship but are not listed. We further confirm that the order of authors listed in the manuscript has been approved by all of us.

The manuscript submitted for publication in BMC Endocrine Disordershas been read and approved by all authors, has not been published, totally or partly, in any other journal even in Persian language and if accepted for publication in this Journal, it will not be published in any other periodicals. Besides, it is confirmed that this article has not been submitted simultaneously to other journals in Iran and/or other countries. I accept the liability of the scientific integrity of the manuscript contents.

We confirm that we have given due consideration to the protection of intellectual property associated with this work and that there are no impediments to publication, including the timing of publication, with respect to intellectual property. In so doing we confirm that we have followed the regulations of our institutions concerning intellectual property.

We understand that the Corresponding Author is the sole contact for the Editorial process (including Editorial Manager and direct communications with the office). She is responsible for communicating with the other authors about progress, submissions of revisions and final approval of proofs.

\section{Availability of data and material}

We have had access to all the data in the study (for original research articles) and accept responsibility for its validity.

\section{Competing interests}

No potential conflicts of interest relevant to this article were reported.

\section{Funding}

This study was approved and funded by the Deputy for Research of Mazandaran University of Medical Sciences (Grant No: 2934). 
Maboudi A. conceived and designed the study, Shafizade S. conceived and designed the study. she also did data collection. Nasiri-Amiri.F, Moaddabi A, Amiri M, Yosefnia-Pasha S reviewed the literature, analyzed and interpreted data, drafted and prepared the manuscript. Qujeq D, Gholami-Bahnemiri M. measured the serum levels of MMP-9 using ELISA kits. Mohammadpour RA. had full access to all the data in the study and takes responsibility for the integrity of the data and the accuracy of the data analysis. The final manuscript was approved by all authors.

\section{Acknowledgement}

This article is extracted from a general dentistry's thesis and is supervised by Mazandaran University of Medical Sciences. Therefore, first of all, I would like to express my gratitude for their financial support. I would also like to sincerely thank all the participants who helped us in this research.

\section{References}

1. Norman R. Dewailly D, Legro RS, Hickey TE. Polycystic ovary syndrome Lancet. 2007; 370:685-97.

2. Fr DD, UK SF, Tarlatzis R. Revised 2003 consensus on diagnostic criteria and long-term health risks related to polycystic ovary syndrome. Fertility and sterility. 2004;81(1).

3. Tehrani FR, Simbar M, Tohidi M, Hosseinpanah F, Azizi F. The prevalence of polycystic ovary syndrome in a community sample of Iranian population: Iranian PCOS prevalence study. Reproductive Biology and Endocrinology. 2011;9(1):39.

4. Azziz R, Marin C, Hoq L, Badamgarav E, Song P. Health care-related economic burden of the polycystic ovary syndrome during the reproductive life span. The Journal of Clinical Endocrinology \& Metabolism. 2005;90(8):4650-8.

5. McCook JG, Reame NE, Thatcher SS. Health-related quality of life issues in women with polycystic ovary syndrome. Journal of Obstetric, Gynecologic, \& Neonatal Nursing. 2005;34(1):12-20.

6. Alberti K, Eckel RH, Grundy SM, Zimmet PZ, Cleeman JI, Donato KA, et al. Harmonizing the metabolic syndrome: a joint interim statement of the international diabetes federation task force on epidemiology and prevention; national heart, lung, and blood institute; American heart association; world heart federation; international atherosclerosis society; and international association for the study of obesity. Circulation. 2009;120(16):1640-5.

7. Elizabeth M, Leslie NS, Critch EA. Managing polycystic ovary syndrome: a cognitive behavioral strategy. Nursing for women's health. 2009;13(4):292-300.

8. Hunter MH, Sterrett JJ. Polycystic ovary syndrome: it's not just infertility. American Family Physician. 2000;62(5):1079-88, 90.

9. Qin JZ, Pang LH, Li MJ, Fan XJ, Huang RD, Chen HY. Obstetric complications in women with polycystic ovary syndrome: a systematic review and meta-analysis. Reproductive Biology and Endocrinology. 2013;11(1):56. 
10. Jones GL, Hall JM, Lashen HL, Balen AH, Ledger WL. Health-Related quality of life among adolescents with polycystic ovary syndrome. Journal of Obstetric, Gynecologic \& Neonatal Nursing. 2011;40(5):577-88.

11. Shen W, Zhang Y, Li W, Cong J, Zhou Y, Ng EH, et al. Effects of tanshinone on hyperandrogenism and the quality of life in women with polycystic ovary syndrome: protocol of a double-blind, placebocontrolled, randomised trial. BMJ open. 2013;3(10):e003646.

12. Azziz R, Woods KS, Reyna R, Key TJ, Knochenhauer ES, Yildiz BO. The prevalence and features of the polycystic ovary syndrome in an unselected population. The Journal of Clinical Endocrinology \& Metabolism. 2004;89(6):2745-9.

13. Revised 2003 consensus on diagnostic criteria and long-term health risks related to polycystic ovary syndrome (PCOS). Human reproduction (Oxford, England). 2004;19(1):41-7.

14. Li Y, Li Y, Yu Ng EH, Stener-Victorin E, Hou L, Wu T, et al. Polycystic ovary syndrome is associated with negatively variable impacts on domains of health-related quality of life: evidence from a metaanalysis. Fertil Steril. 2011;96(2):452-8.

15. Williams RC. Periodontal disease. New England Journal of Medicine. 1990;322(6):373-82.

16. Javed F, Ahmed A. Proinflammatory cytokines in the saliva, gingival crevicular fluid and serum of diabetic patients with periodontal disease. J Res Pract Dent. 2013;2013:1-10.

17. Akcalı A, Bostanci N, Özçaka Ö, Öztürk-Ceyhan B, Gümüş P, Tervahartiala T, et al. Elevated matrix metalloproteinase-8 in saliva and serum in polycystic ovary syndrome and association with gingival inflammation. Innate immunity. 2015;21(6):619-25.

18. Kinane D, Bouchard P, Periodontology GEotEWo. Periodontal diseases and health: consensus report of the sixth European workshop on periodontology. Journal of clinical periodontology. 2008;35:3337 .

19. Akcalı A, Bostanci N, Özçaka Ö, Öztürk-Ceyhan B, Gümüş P, Buduneli N, et al. Association between polycystic ovary syndrome, oral microbiota and systemic antibody responses. PLoS One. 2014;9(9):e108074.

20. Victor VM, Rovira-Llopis S, Bañuls C, Diaz-Morales N, de Marañon AM, Rios-Navarro C, et al. Insulin resistance in PCOS patients enhances oxidative stress and leukocyte adhesion: role of myeloperoxidase. PLoS One. 2016;11(3):e0151960.

21. Pawelczak M, Rosenthal J, Milla S, Liu Y-H, Shah B. Evaluation of the pro-inflammatory cytokine tumor necrosis factor-a in adolescents with polycystic ovary syndrome. Journal of pediatric and adolescent gynecology. 2014;27(6):356-9.

22. Souza dos Santos AC, Soares NP, Costa EC, de Sá JCF, Azevedo GD, Lemos TMAM. The impact of body mass on inflammatory markers and insulin resistance in polycystic ovary syndrome. Gynecological Endocrinology. 2015;31(3):225-8.

23. Rahiminejad ME, Moaddab A, Zaryoun H, Rabiee S, Moaddab A, Khodadoustan A. Comparison of prevalence of periodontal disease in women with polycystic ovary syndrome and healthy controls. Dental research journal. 2015;12(6):507. 
24. Kellesarian S, Malignaggi V, Kellesarian T, Al-Kheraif A, Alwageet M, Malmstrom H, et al. Association between periodontal disease and polycystic ovary syndrome: a systematic review. International journal of impotence research. 2017;29(3):89.

25. Akcalı A, Bostanci N, Özçaka Ö, Gümüş P, Öztürk-Ceyhan B, Tervahartiala T, et al. Gingival Inflammation and Salivary or Serum Granulocyte-Secreted Enzymes in Patients With Polycystic Ovary Syndrome. Journal of periodontology. 2017;88(11):1145-52.

26. Dursun E, Akalin FA, Guncu GN, Cinar N, Aksoy DY, Tozum TF, et al. Periodontal disease in polycystic ovary syndrome. Fertil Steril. 2011;95(1):320-3.

27. Hajishengallis G. Periodontitis: from microbial immune subversion to systemic inflammation. Nature Reviews Immunology. 2015;15(1):30-44.

28. Navazesh M. Methods for collecting saliva. Annals of the New York Academy of Sciences. 1993;694(1):72-7.

29. Tanguturi SC, Nagarakanti S. Polycystic ovary syndrome and periodontal disease: underlying links-a review. Indian journal of endocrinology and metabolism. 2018;22(2):267.

30. Lam OL, Zhang W, Samaranayake LP, Li LS, McGrath C. A systematic review of the effectiveness of oral health promotion activities among patients with cardiovascular disease. International journal of cardiology. 2011;151(3):261-7.

31. Amiri AA, Maboudi A, Bahar A, Farokhfar A, Daneshvar F, Khoshgoeian HR, et al. Relationship between type 2 diabetic retinopathy and periodontal disease in Iranian adults. North American journal of medical sciences. 2014;6(3):139.

32. Slowik J, Wnuk MA, Grzech K, Golenia A, Turaj W, Ferens A, et al. Periodontitis affects neurological deficit in acute stroke. Journal of the neurological sciences. 2010;297(1-2):82-4.

33. Wang Z, Zhou X, Zhang J, Zhang L, Song Y, Hu FB, et al. Periodontal health, oral health behaviours, and chronic obstructive pulmonary disease. Journal of clinical periodontology. 2009;36(9):750-5.

34. Kelly CC, Lyall H, Petrie JR, Gould GW, Connell JM, Sattar N. Low grade chronic inflammation in women with polycystic ovarian syndrome. The Journal of Clinical Endocrinology \& Metabolism. 2001;86(6):2453-5.

35. Ozcaka O, Ceyhan ÖB, Akcali A, BiÇakci N, Lappin D, Buduneli N. is there an Interaction between Polycystic Ovary Syndrome and Gingival Inflammation?: p0113. Journal of Clinical Periodontology. 2012;39:111.

36. Mohlig M, Spranger J, Osterhoff M, Ristow M, Pfeiffer A, Schill T, et al. The polycystic ovary syndrome per se is not associated with increased chronic inflammation. European Journal of Endocrinology. 2004;150(4):525-32.

37. Rudnicka E, Kunicki M, Suchta K, Machura P, Grymowicz M, Smolarczyk R. Inflammatory Markers in Women with Polycystic Ovary Syndrome. BioMed Research International. 2020;2020.

38. Arpaci H. An evaluation of new and current inflammatory markers in patients with polycystic ovary syndrome. Annals of Medical Research. 2020;27(1):1-6. 
39. Ramamoorthy S, Bhuvaneswari K. A Cross Sectional Study on the Status of Inflammatory Markers in Polycystic Ovary Syndrome (Pcos) in Indian Population. Biomedical and Pharmacology Journal. 2019;12(4):1975-83.

40. Duleba AJ, Dokras A. Is PCOS an inflammatory process? Fertility and sterility. 2012;97(1):7-12.

41. Liu B, Guan YM, Zheng Jh. Elevated serum levels of matrix metalloproteinase-2 in women with polycystic ovarian syndrome. International Journal of Gynecology \& Obstetrics. 2007;96(3):204-5.

42. Ranjbaran J, Farimani M, Tavilani H, Ghorbani M, Karimi J, Poormonsefi F, et al. Matrix metalloproteinases 2 and 9 and MMP9/NGAL complex activity in women with PCOS. Reproduction. 2016;151(4):305-11.

43. Dambala K, Vavilis D, Bili E, Goulis DG, Tarlatzis BC. Serum visfatin, vascular endothelial growth factor and matrix metalloproteinase-9 in women with polycystic ovary syndrome. Gynecological Endocrinology.2017;33(7):529-33

44. Gerlach RF, Uzuelli JA, Souza-Tarla CD, Tanus-Santos JE. Effect of anticoagulants on the determination of plasma matrix metalloproteinase (MMP)-2 and MMP-9 activities. Analytical biochemistry. 2005;344(1):147-9.

45. Gerlach RF, Demacq C, Jung K, Tanus-Santos JE. Rapid separation of serum does not avoid artificially higher matrix metalloproteinase (MMP)-9 levels in serum versus plasma. Clinical biochemistry. 2007;40(1-2):119-23.

46. Souza-Tarla CD, Uzuelli JA, Machado AA, Gerlach RF, Tanus-Santos JE. Methodological issues affecting the determination of plasma matrix metalloproteinase (MMP)-2 and MMP-9 activities. Clinical biochemistry. 2005;38(5):410-4.

47. Visse R, Nagase H. Matrix metalloproteinases and tissue inhibitors of metalloproteinases: structure, function, and biochemistry. Circulation research. 2003;92(8):827-39.

48. Kessenbrock K, Plaks V, Werb Z. Matrix metalloproteinases: regulators of the tumor microenvironment. Cell. 2010;141(1):52-67.

49. Wotherspoon AC, Diss T, Pan L, Isaacson P, Doglioni C, Moschini A, et al. Regression of primary lowgrade B-cell gastric lymphoma of mucosa-associated lymphoid tissue type after eradication of Helicobacter pylori. The Lancet. 1993;342(8871):575-7.

50. Stetler-Stevenson WG. Type IV collagenases in tumor invasion and metastasis. Cancer and Metastasis Reviews. 1990;9(4):289-303. 